\title{
WRTS: Wireless Sensor Based Real Time Traffic Information System
}

\author{
G.Bhaskar \\ Department of Computer Science and Engineering \\ Siddaganga Institute of Technology, \\ Tumkur, Karnataka, India.
}

\author{
Raghu BA, \\ Department of Computer Science and Engineering \\ Siddaganga Institute of Technology, \\ Tumkur, Karnataka, India.
}

\begin{abstract}
Due to increasing traffic density, driving takes longer time within cities than in highways. The situation is worse in peak hours, when people travel from home to offices and vice versa. Emergency services, like ambulance services, fire brigade, police service etc., are directly affected due to increased traffic density. The existing traffic monitoring techniques will fail to provide real time traffic information to the user. India is known for billion populations and like in majority of developing countries like Indian roads are non-lane based and it has chaotic traffic. The existing traffic monitoring techniques are mainly infrastructure based system. Which is expensive and it requires constant maintenance. This paper focuses on fetching real time traffic information which is infrastructure less based system which is inexpensive in nature and it is also reachable to a normal person, like fetching real time traffic information from user who carry smart phones which play a role of sensor during their travel. This leads to building of wireless sensor network of mobiles.
\end{abstract}

Keywords: Sensing; BTS, GPS, Traffic Light control, Chaotic Traffic, WRTS;

\section{INTRODUCTION}

Road traffic congestion is a recurring problem worldwide. In developing countries like India, a fast growing economy, the problem is acutely felt in almost all major cities. This is primarily because infrastructure growth is slow compared to growth in number of vehicles, due to space and cost constraints. Secondly, like in majority of developing countries like India traffic being non-lane based and chaotic is largely different from the western traffic. Like India, the second most populous country in the world, and a fast growing economy, is seeing terrible road congestion problems in its cities. Building infrastructure, levying proper taxes to curb private vehicle growth and improving public transport facilities are long-term solutions to this problem. These permanent solution approaches need government intervention. The Government of India has committed Rs.234,000 corers in the urban infrastructure sector Bus Rapid Transit (BRT), metro rails and mono rails are being built in different cities to encourage the use of public transport[10]. But still there is a steep growth of private vehicles Some cities like Bangalore, Pune, Hyderabad Delhi-NCR, with their sudden growths in the IT sector, also have a steep growth in population, further increasing transportation needs. Meeting such growth with infrastructure growth is seemingly infeasible, primarily because of space and cost constraints. Intelligent management of traffic flows and making commuters more informed about traffic and road status, can reduce the negative impact of congestion, though cannot solve it altogether. Traffic monitoring systems deployed until now, utilize data collected mainly through fixed sensors, which provide information about the number and speed of vehicles that cross them. Nevertheless, this type of system is not deployed at wide scales mostly because of its high cost. Mobile devices used as traffic sensors present major advantages compared to other solutions[11]. So far, all the existing solutions require the design, implementation and maintenance of special infrastructure, which can be both expensive and difficult to deploy. On the other hand, cellular networks are already widely deployed and provide large population coverage. Modern mobile devices come equipped with Global Position System (GPS) receivers which can calculate the device's location and speed with sufficient accuracy. On the communication side, many wireless standards (3G/4G/WLAN) are already included and most of the upcoming standards are expected to be integrated in the near future. This connectivity capacity and the array of sensors that mobile devices are equipped with, make them suitable for gathering a great amount of traffic data. Finally, modern mobile devices feature high resolution screens or touch screens that can be used for interacting with the driver and display detailed information. This paper presents Wireless Sensor Network Based Real Time Traffic Information System (WRTS) this focuses on fetching real time traffic information from user who carry smart phones which play a role of sensor during their travel. This leads to building of wireless sensor network of mobiles. This paper is organised as follows. In Section II of the paper, we present the Related Work, Section III gives the Scenario and Proposed system, and in section IV Implementation and results are presented, and section $\mathrm{V}$ deals with Conclusion, VI is Acknowledgement and finally Section VII have References for the paper.

\section{RELEATED WORK 2.1 Impact of Traffic Jam in India}

As the economic gloom intensifies, a report by the Ministry of Road, Transport and Highways only makes the picture worse. Traffic snarls on highways, delays at toll plazas, 
accidents and political blockades annually cost the economy nearly $\boldsymbol{R} \boldsymbol{s . 4 0}$ billion in lost truck-operating hours, says the Second Report on Operational Efficiency of Freight Transportation by Road in India, released by minister of road, transport and highways C.P. Joshi here on July $1^{\text {st }} 2012[1]$.

Traffic jam is a condition on road networks that occurs as use increases, and is characterized by slower speeds, longer trip times, and increased vehicular queuing. As demand approaches the capacity of a road (or of the intersections along the road), extreme traffic jam sets in. When vehicles are fully stopped for periods of time, this is colloquially known as a traffic jam.

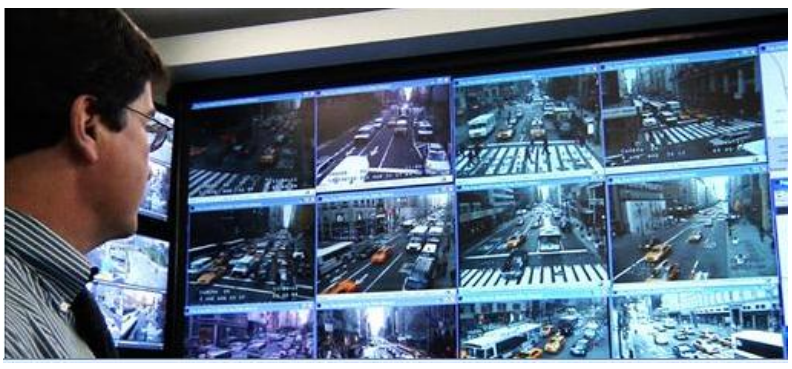

Fig 2.1: CCTV View of Traffic jams in Bangalore.

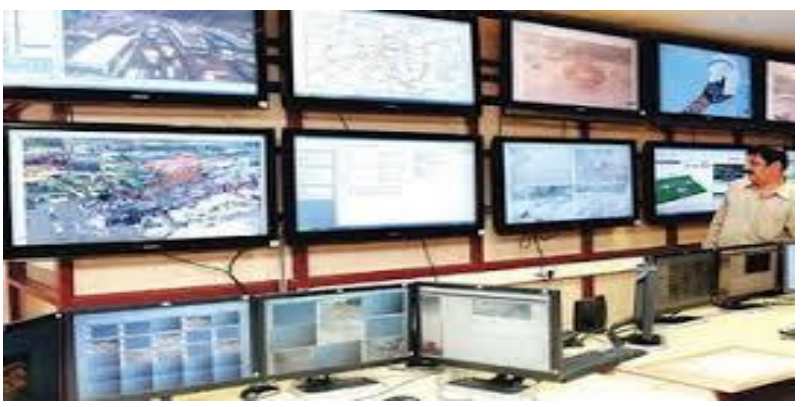

Fig 2.2: Bangalore Traffic control Room.

Traffic jam has a number of negative effects:

- Wasting time of motorists and passengers. As a non-productive activity for most people, traffic jam reduces regional economic health.

- Delays, which may result in late arrival for employment, meetings, and education, resulting in lost business, disciplinary action or other personal losses.

- Inability to forecast travel time accurately, leading to drivers allocating more time to travel "just in case", and less time on productive activities.

- Emergencies: blocked traffic may interfere with the passage of emergency vehicles traveling to their destinations where they are urgently needed.
- Higher chance of jam due to tight spacing and constant stopping-and-going.

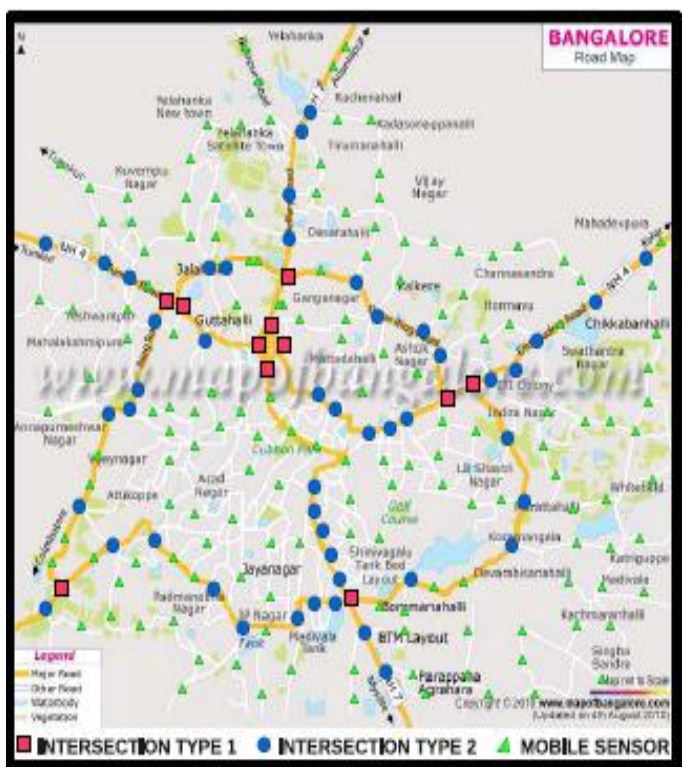

Fig 2.3: Bangalore City wide sensing Architecture [4]

There are two main kinds of roads, the yellow lines denote the major roads and the white lines denote minor roads. The junctions where the major roads intersect one another are shown by red squares and those where the minor roads intersect the major roads are shown by blue dots. Traffic light control is a vital necessity for both these type of intersections that will govern the traffic flow pattern over the entire city. Currently, in absence of automated signal control techniques, the traffic lights are either statically calibrated, or controlled by on-road policemen, or remotely controlled by manually seeing video feeds at the traffic control room.

As for like Indian commuters, the traffic applications currently available are rudimentary, with periodic updates gathered from traffic police broadcast on FM radios. There are a few route planners that give bus and train plans, but the plans are static, without considering the current congestion levels on different roads.

\section{Choice of sensing method}

Participatory sensing data inherently noisy [3]. Also probe vehicles might not be present at a given intersection at all times. Such sensing methods can thus be used for applications like travel time estimates and congestion maps to be disseminated to commuters, which can tolerate aperiodicity and noisiness. Applications like traffic light control on the other hand, need dedicated static sensors on the road that give highly accurate and strictly periodic updates about traffic conditions. Static sensors are costly but accurate, mobile and hybrid sensors are cheap but 
noisy. Based on applications, we need to make a careful choice of which mode to use when and how to mix them appropriately [3]. The red squares and blue dots in Fig. 2.3 would thus be probable candidates for static sensing, while the mobile and hybrid sensors would span the city, in varying densities over time, as the green triangles in the figure.

\subsection{Sample Case: Bangalore Traffic Monitoring System (BTS)}

Explaining the existing system in the context of (BTS) At present, out of 160 cameras in Bangalore, connected to central traffic control room by BSNL leased lines, at most 90 cameras send data at any given time, because of problems in the wired connections. Instead of this star topology, will a tiered architecture help, where video feeds from a subset of roads are processed at local control rooms, before the information is passed to central control? Should there be a mix of wired and wireless communication to reduce cost and overhead of laying copper, or will wireless be too unreliable? Can communication costs be reduced through in-field computation and data compression? As for mobile sensing models, quantitative analysis of $3 \mathrm{G}$ and GPRS performance on Indian roads, using mobile for traffic data upload and download would be a feasible solution.

\section{SCENARIO AND PROPOSED SYSTEM}

This paper deals with scenario of tracking and fetching real time traffic information from the user's mobile during their travel. Application will update this traffic information to all the users in the same path. In this proposed system we have two algorithms Traffic_ServerRun and Traffic_ClientRun which usually runs on server and client which is user's mobile.

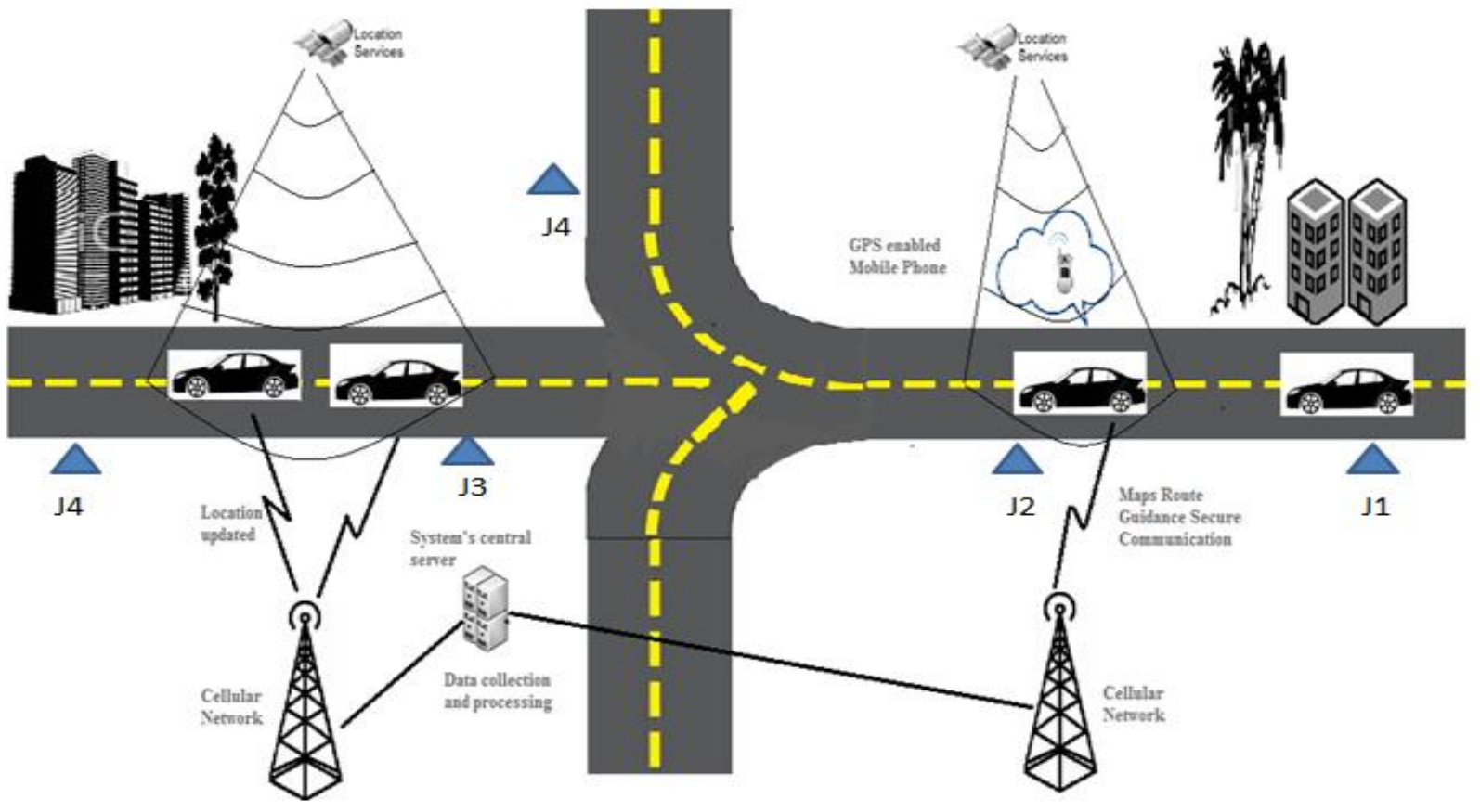

Figure 1: Illustrating complete Scenario of WRTS 


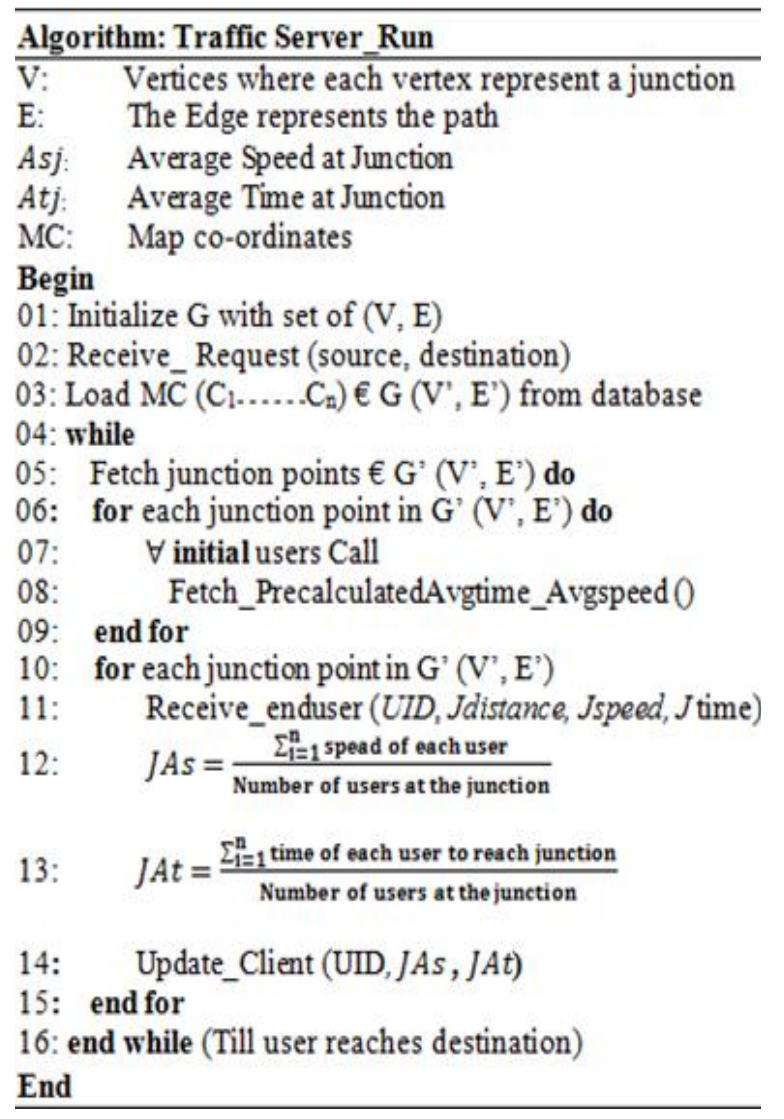

Algorithm: Traffic Client_Run

Input: Sensor Network G (V, E), Source, Destination

Output: Real time traffic information for user to reach destination

$\mathrm{T}[\mathrm{Lt}, \mathrm{Ln}]: \quad$ Object Trajectory Data

Junction List[i]: Vector of Junction

Pjt: $\quad$ Previous Junction Time

Cjt: $\quad$ Current Junction Time

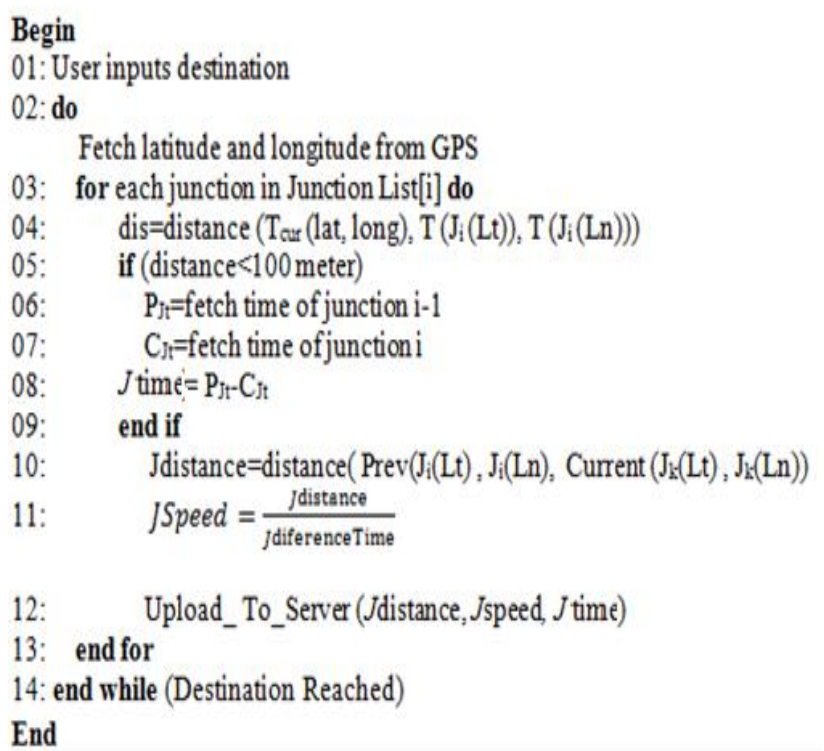

Begin

01: User inputs destination

Fetch latitude and longitude from GPS

03 : for eachuntion in Jantion Lis([)

04. dis=distance $\left(\mathrm{T}_{\text {eut }}\right.$ (lat, long), $\mathrm{T}\left(\mathrm{J}_{\mathrm{i}}(\mathrm{Lt} \mathrm{t}), \mathrm{T}\left(\mathrm{J}_{\mathrm{i}}(\mathrm{Ln})\right)\right)$

05: if (distance $<100$ meter)

06: $\quad P_{\mathrm{t} t}=$ fetch time of junction $\mathrm{i}-1$

07: $\quad \mathrm{Cl}_{\mathrm{f}}=$ fetch time of junction

08: $J$ time $=\mathrm{P}_{\mathrm{Pt}} \mathrm{CH}_{\mathrm{t}}$

09: $\quad$ end if
11: $\quad$ JSpeed $=\frac{\text { Jdistance }}{\text { Jdiference Time }}$
12: Upload_To_Server (Jdistance, Jspeed, J time)
14: end while (Destination Reached)
End

10.

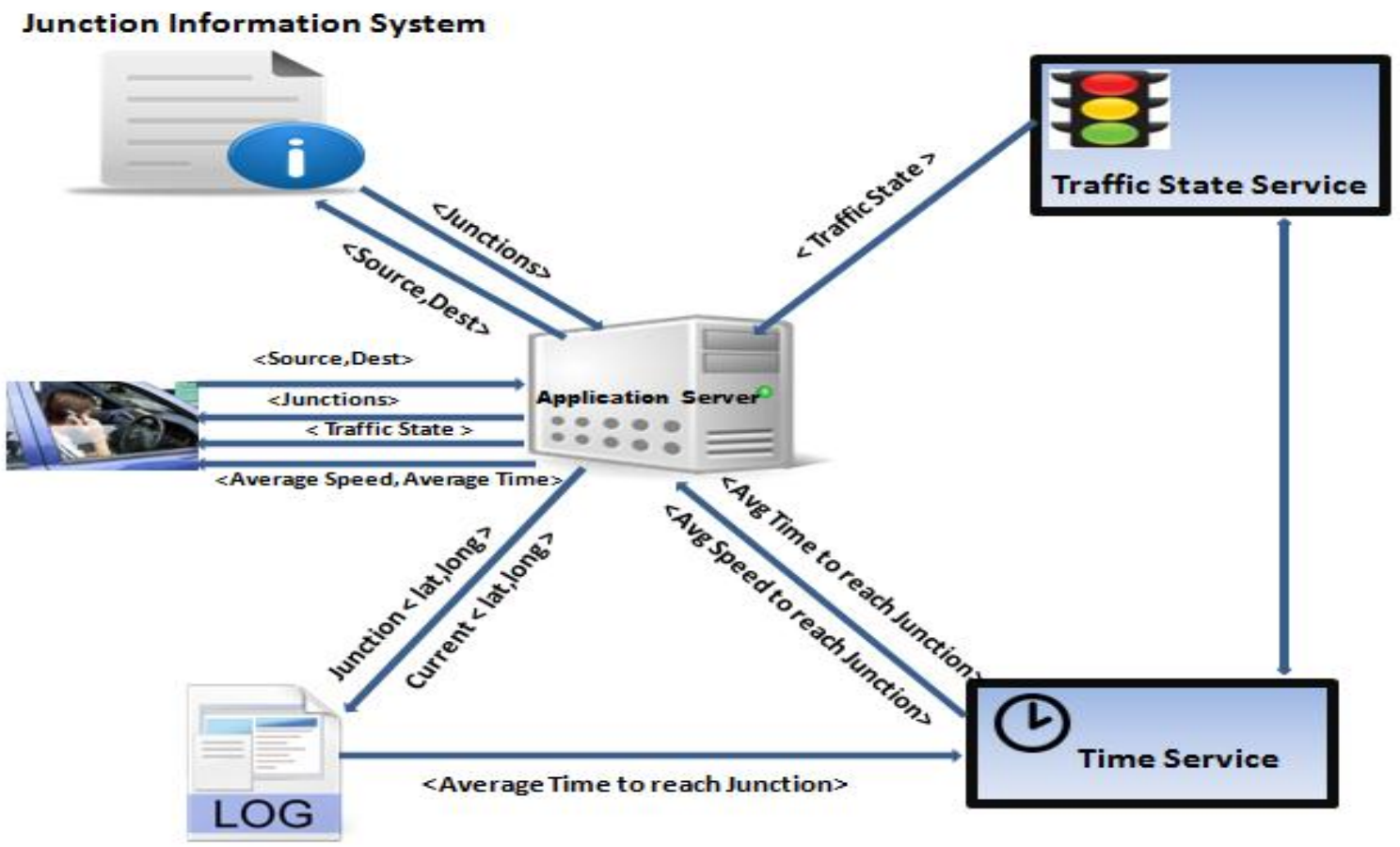

Figure 2: Framework/Architecture of Real Time Information System 
Application is initiated once users starts journey by entering source and destination when there is a connection with the GPS service and the coordinates of the device are calculated, it notifies the communication thread to send updates to the server. This thread is running continuously on the background and calculates the location coordinates, Junction speed, Junction time of a user and constantly updates the server. In the meanwhile, the control thread is notified and a new map thread is initiated with focus on the mobile device's position. The map thread, using the Google Map API, is connected to a map server and presents the map of the area on the screen. The server application is listening on a specified port and is responsible for starting a serving thread for each connected client. Location updates and traffic updates are collected from all the clients, and the server sends back with Average speed, Average time to reach a junction to all the user on the same path. The log files with the client's tracks are stored on the server for further processing regarding the development of a traffic reconstruction algorithm As illustrated in Fig 1 and Fig 2 which explains the overall working of Wireless Sensor based Real Time Traffic Information System architecture. user gets traffic updates periodically before user reaches next junction in the path. For testing purposes, an application, which can emulate multiple clients by reading tracks location coordinates, calculating average speed, average time constantly and updating the same to all the users in the path this scenario is explained with snap shots in Implementation and Results section. The current implementation of secure communications between client and server is based on an already well tested protocol as SSL, ensuring the confidentiality of the communication and the proper authentication of the involved parties. For an application deployed at large scale, it has the drawback of having to issue a separate digital certificate for each user.

\section{IMPLEMENTATION AND RESULTS}

The core of the system is the mobile application running on the mobile devices. Application had been developed and implemented on android platform it uses Google Api for displaying maps. Application has two software threads

User Interface Functionality: Responsible for user interface the interaction with the user and control of the application.

Map and Traffic Functionality: Responsible for displaying map with traffic information based on the information sent from the server.

\subsection{RESULTS}

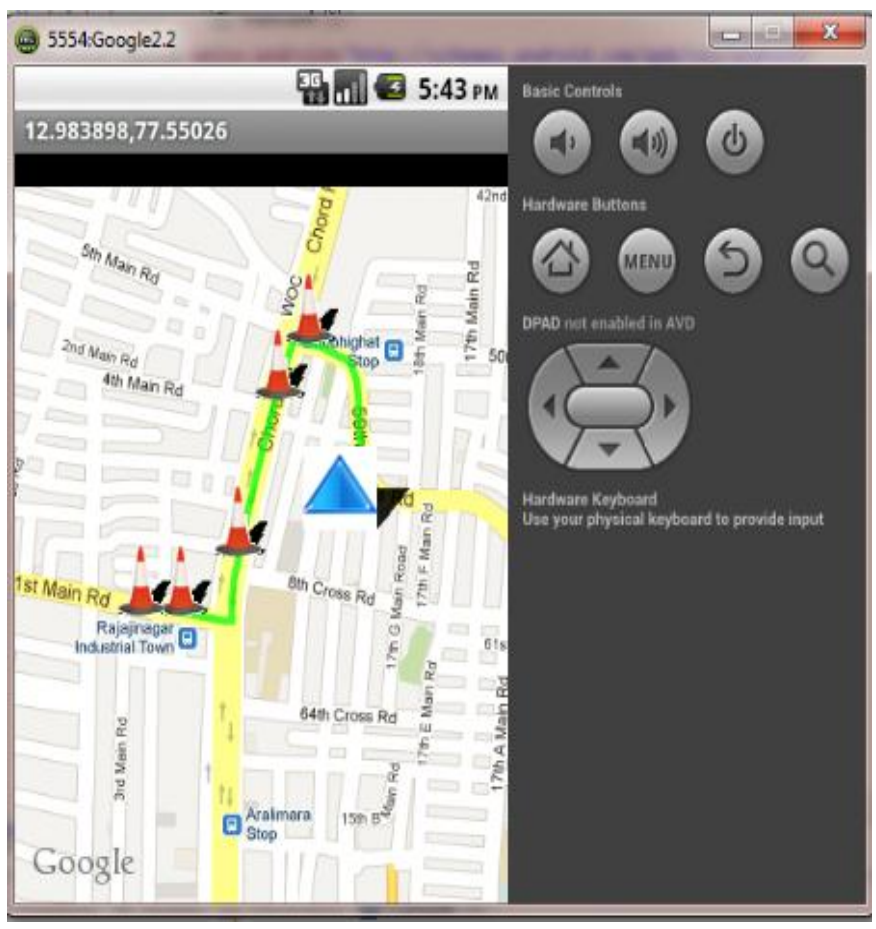

Figure 3:Illustrating junctions between source(Kormangala) and destination(Rajajinagar).

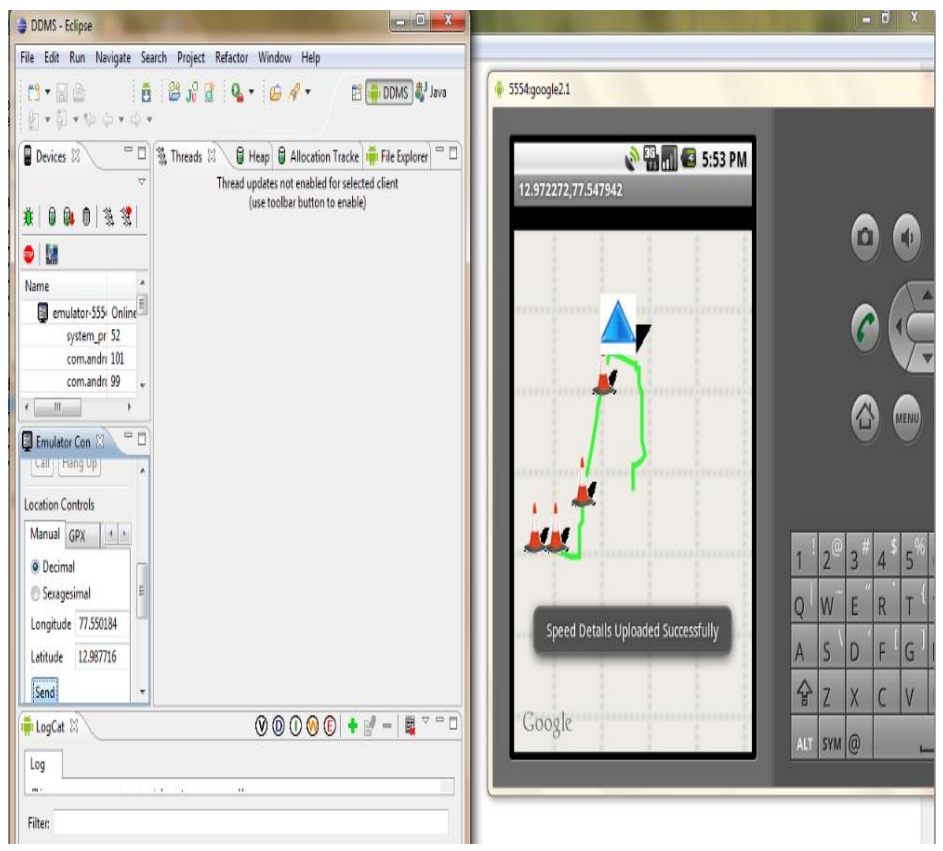

Figure 4: As user A moves to next junction his speed, time, location information is updated to server. 


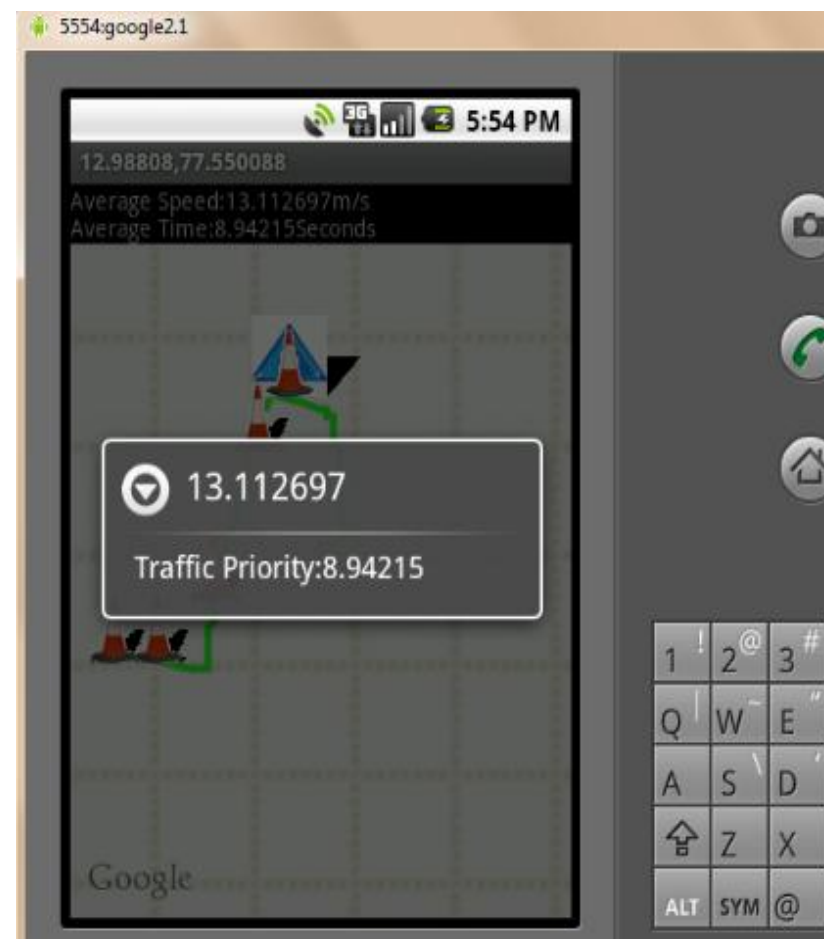

Figure 5: Illustrating Average speed and Average time (traffic priority).

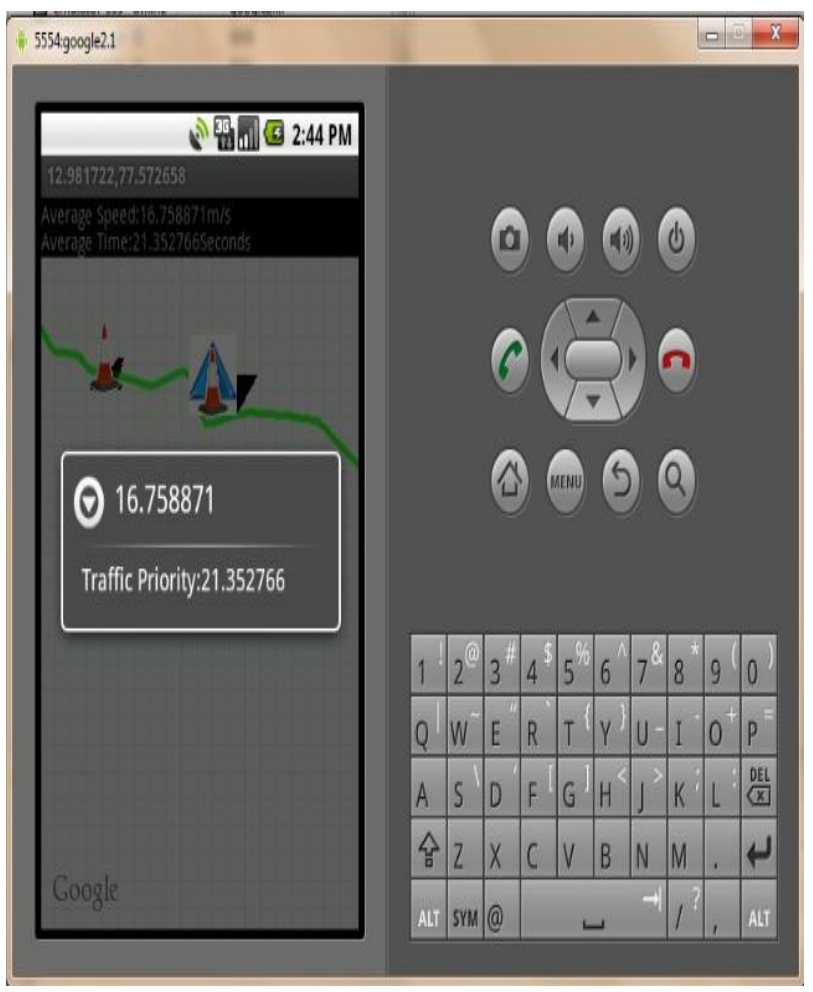

Figure 6:Illustrating another user B as he moves to another junction he gets calculated Average speed and Average time from server based on the other user(A) who had travelled in that path.

\section{CONCLUSION}

This paper Presents Wireless sensor based Real time traffic Information System where mobile is used as sensor to capture traffic information which will overcome the existing Traffic Monitoring Techniques. This system will reduce Infrastructure cost and Monitoring cost of static deployed sensors in roads, because system WRTS uses mobile as sensors which will lead to building of Mobile wireless sensor network. This system is user friendly and easy to use. This system would be useful in reducing traffic jams.

\section{ACKNOWLEDGMENT}

I would like to acknowledge our college Siddaganga Institute of Technology, parents, I thank my advisor Asst Prof.G. Bhaskar for not only providing ideas and guidance but also for working along with me and being moral support for me.

\section{REFERENCES}

[1] Traffic Safety and City Public Transport System:

Case Study of Bengaluru, India P. S. Kharola, Geetam Tiwari, and Dinesh Mohan

[2] 3GPP TS 25.305, Stage 2 Functional Specification of UE Positioning, Dec. 2009.

[3] Intelligent Transport Systems for Indian Cities

Rijurekha Sen, Bhaskaran Raman

[4] http://www.mapofbangalore.com/maps/bangalore-roadmap.html.

[5] M. El Najjar and P. Bonnifait, "A road-matching method for precise vehicle localization using belief theory and Kalman filtering", Autonomous Robots, vol. 19, 2005, pp. 173-191.

[6] Y. Wang and M. Papageorgiou, "Real-time freeway traffic state estimation based on extended Kalman filter: a general approach", Transportation Research Part B: Methodological, vol. 39, 2005, pp. 141-167.

[7] L. Mihaylova and R. Boel, "A particle filter for freeway traffic estimation", 43rd IEEE Conference on Decision and Control, 2004, pp. 2106-2111.

[8] S. Pramanik, "An efficient path computation model for hierarchically structured topographical road maps", IEEE Transactions on Knowledge and Data Engineering, vol. 14, 2002, pp. 1029-1046.

[09] GPS tracking unit Wikipedia The free encyclopedia available http://en.wikipedia.org/wiki/GPS_tracking_unit.

[10] Traffic congestion in Indian cities: Challenges of arising power Azeem Uddin Draft, 50th IEEE Conference on Decision and Control, 2004, pp. 2106-2111.

[11]TheMobileMillenniumProject.http://traffic.berkeley.ed $\mathrm{u} /$ 